\title{
Spectral dynamics of linearly polarized bright exciton in InP/ZnSe colloidal quantum dots
}

Spectroscopy of single colloidal quantum dots (QDs), especially at cryogenic temperature, helps to understand the inherent properties of nanocrystals that are often hidden in ensemble level studies. This applies in particular to InP-based QDs, which attract increasing interest as $\mathrm{Cd}$-free alternative nanocrystals yet were hardly investigated at the single $Q D$ level. Here, we discuss the photoluminescence properties of single InP/ZnSe QDs, both at room temperature and at cryogenic temperature. While ensemble level measurements feature a luminescent linewidth of around $50 \mathrm{~nm}$, we find that emission spectrum of single $\mathrm{InP} / \mathrm{ZnSe}$ QDs can have a linewidth as narrow as $14 \mathrm{~nm}(50 \mathrm{meV})$. Hence, the relatively broad emission line that characterizes ensembles of InP-based QDs is by no means an intrinsic material property. In addition, we found that InP/ZnSe QDs combine a nearly blinking free emission with a high purity single photon emission $\left(g^{2}(0)<0.03\right)$, also well beyond the saturation intensity.[1] Cryogenic single QD spectra, on the other hand, consist of zero-phonon lines that can be as narrow as $40 \mu \mathrm{eV}$. Polarization resolved spectra point to a linearly polarized spectral doublet from the bright exciton. At lower excitation intensities, jitter was negligible and spectra could be integrated for tens of seconds without erasing the doublet splitting of $1.2 \mathrm{meV}$. At higher excitation intensities, jitter becomes more severe and switching between emission from the exciton-doublet and the trion-singlet can be observed. This indicates that spectral jitter finds its origin in changes of the local electric field caused by the temporal trapping of one charge carrier. In summary, we find conclude that single InP QDs have emission characteristics similar to the extensively studied CdSe-based QDs.

Moreover, the narrow emission lines, limited jitter and fluorescence intermittency of single InP/ZnSe QDs holds great promise to further explore these materials as a solutionprocessable single-photon emitter and improve the ensemble level characteristics of these materials.

\section{Reference:}

[1] Chandrasekaran, V.; Tessier, M. D.; Dupont, D.; Geiregat, P.; Hens, Z.; Brainis, E. Nano Letters 2017, 17 (10), 6104-6109. 